\title{
Atomic Bases and T-path Formula for Cluster Algebras of Type $D$
}

\author{
Emily Gunawan $\|^{\dagger}$ and Gregg Musiker|" \\ School of Mathematics, University of Minnesota, Minneapolis, MN 55455, USA
}

\begin{abstract}
We extend a $T$-path expansion formula for arcs on an unpunctured surface to the case of arcs on a oncepunctured polygon and use this formula to give a combinatorial proof that cluster monomials form the atomic basis of a cluster algebra of type $D$.

Résumé. Nous généralisons une formule de développement en T-chemins pour les arcs sur une surface non-perforée aux arcs sur un polygone à une perforation. Nous utilisons cette formule pour donner une preuve combinatoire du fait que les monômes amassées constituent la base atomique d'une algèbre amassée de type $D$.
\end{abstract}

Keywords: cluster algebra, triangulated surface, atomic basis

\section{Introduction}

Cluster algebras, introduced by Fomin and Zelevinsky [FZ02] in 2000, are commutative algebras equipped with a distinguished set of generators, called cluster variables, which can be constructed recursively starting from an initial set of $n$ cluster variables. We are interested in cluster algebras arising from bordered surfaces with marked points [FG06, FG09, GSV05, FST08]. In particular, we study cluster algebras of type $D_{n}$ (type $D$ for short), which are of finite type (as classified in [FZ03]) and correspond to oncepunctured $n$-gons, as also described in detail in [Sch08]. Other, related work on type $D$ cluster algebra combinatorial models include [FZ03, BM09, CP14].

Our first result is a Laurent polynomial expansion formula for cluster variables arising from a oncepunctured polygon in terms of certain paths (called $T^{o}$-paths) on an ideal triangulation $T^{o}$ of the surface. This is an extension of the $T$-path formula which is given for any unpunctured surface by Schiffler and Thomas [ST09, Sch10]. Our proof takes advantage of two facts proven by the second author, Schiffler, and Williams: (1) a Laurent polynomial expansion formula for cluster variables arising from any surface in terms of perfect matchings of a snake graph [MSW11], and (2) a bijection between these perfect matchings and $T$-paths arising from any unpunctured surface [MS10]. The motivation behind having a $T^{o}$-path formula for type $D$ is discussed in the next paragraph.

\footnotetext{
${ }^{\dagger}$ Email: egunawan@umn. edu. Supported by NSF Grants DMS-1067183 and DMS-1148634.

‡Email: musiker@umn. edu. Supported by NSF Grants DMS-1067183 and DMS-1148634. 
Our second result is a specific case of a result of [Cer11, CL12], proven by representation theoretic methods, that the basis consisting of all cluster monomials is in fact the atomic basis for any skewsymmetric cluster algebra of finite type (see Section 4.1). We give a combinatorial proof of this fact for coefficient-free cluster algebras of type $D$. Our proof relies heavily on the $T^{\circ}$-path formula for type $D$ and is inspired by Dupont and Thomas' work in [DT13] on atomic bases for cluster algebras of type $A$ and $\widetilde{A}$.

In Section 2, we provide background material on ideal triangulations and tagged triangulations, focusing on the case of once-punctured polygons. Section 3 presents our first result (Theorem 3.11), an extension of the $T$-path formula of [Sch10, ST09] to once-punctured polygons. Finally, in Section 4, we give our second and main result (Lemma 4.5), which is a combinatorial proof, using the $T$-path formula, that the cluster monomials form the atomic basis for any type $D$ cluster algebra. The proofs of both results are available in [GM14].

\section{Background: Cluster algebras arising from once-punctured disks}

For the reader's convenience, we begin by reviewing terminology arising in the theory of cluster algebras from marked surfaces from [FST08, Sections 2 and 7]. We restrict our attention to the case of a oncepunctured polygon, which often simplifies the notation. Let $\mathcal{C}_{n}$ denote a once-punctured $n$-gon, i.e. a disk with a marked point (called the puncture) in the interior and $n$ marked points on the boundary.

Definition 2.1 (Ordinary arcs) $A$ boundary edge of $\mathcal{C}_{n}$ is a segment of the boundary between two consecutive boundary marked points. An ordinary arc $\gamma$ of $\mathcal{C}_{n}$ is a curve (considered up to isotopy) in $\mathcal{C}_{n}$ such that the endpoints of $\gamma$ are marked points, $\gamma$ does not cross itself except possibly at its endpoints, $\gamma$ does not cross the boundary of $\mathcal{C}_{n}$ except possibly at its endpoints, and $\gamma$ is not contractible to a marked point or homotopic to a boundary edge.

$A$ radius is an arc between a boundary marked point and the puncture. Following [DT13], a peripheral arc is an arc with both endpoints on the boundary. An $\ell$-loop is a loop cutting out a once-punctured monogon with a sole puncture inside it. An $\ell$-loop is considered a peripheral, ordinary arc.

Definition 2.2 (Compatibility of ordinary arcs, ideal triangulations) Two distinct ordinary arcs are said to be compatible if they do not intersect except possibly at endpoints. Also, each arc is compatible with itself. A maximal (by inclusion) collection of distinct, pairwise compatible ordinary arcs is called an ideal triangulation. The ordinary arcs of an ideal triangulation cut the surface into ideal triangles. We call an $\ell$-loop and the radius it encloses a self-folded triangle.

Definition 2.3 (Tagged arcs) $A$ tagged arc of $\mathcal{C}_{n}$ is obtained by marking ("tagging") each endpoint of an ordinary arc (that is not an $\ell$-loop) $\beta$ either plain or notched such that the endpoints of $\beta$ on the boundary must be tagged plain. A notched tagging is indicated by a bow tie (see Figure $1(a)$ ). Note that a tagged arc never cuts out a once-punctured monogon, i.e. an $\ell$-loop is not a tagged arc.

Definition 2.4 (Compatibility of tagged arcs, tagged triangulations of $\mathcal{C}_{n}$ ) The following is a complete list of possible compatible pairs $\{\alpha, \beta\}$ of tagged arcs of $\mathcal{C}_{n}$. Note that every endpoint on the boundary must be tagged plain.

- $\alpha$ and $\beta$ are two peripheral arcs that do not intersect in the interior of $\mathcal{C}_{n}$.

- $\alpha$ and $\beta$ are a peripheral arc and a radius that do not intersect in the interior of $\mathcal{C}_{n}$. 
- $\alpha$ and $\beta$ are two radii both adjacent to the same boundary marked point (tagged plain) but $\alpha$ is tagged plain at the puncture and $\beta$ is tagged notched at the puncture.

- $\alpha$ and $\beta$ are two radii with distinct boundary endpoints (tagged plain at boundary endpoints) and tagged the same way at the puncture.

- $\alpha$ and $\beta$ are equal.

A maximal (by inclusion) collection of distinct, pairwise compatible tagged arcs is called a tagged triangulation. A collection of pairwise compatible tagged arcs (considered with multiplicity) is called a multi-tagged triangulation.

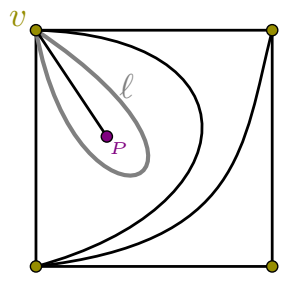

(a) An ideal triangulation $T^{o}$ and the corresponding tagged triangulation $T=\iota\left(T^{o}\right)$.

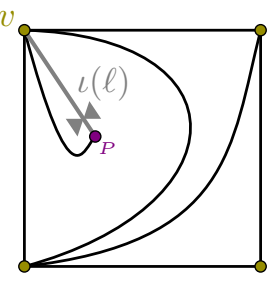

는

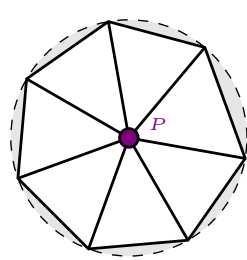

b) Wheel-like triangulation around the puncture.

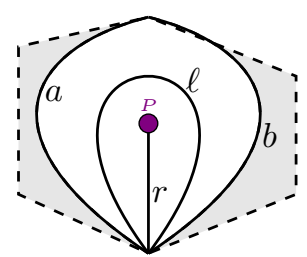

(c) A self-folded triangle around the puncture where $a, b$ are peripheral arcs or boundary edges.

Fig. 1: Ideal triangulations of a once-punctured polygon.

Definition 2.5 (Representing ordinary arcs as tagged arcs) Any ordinary arc $\beta$ can be represented by a tagged arc $\iota(\beta)$ as follows. Suppose $\beta$ is an $\ell$-loop (based at marked point $v$ ) which encloses a radius $r$, where $r$ is the unique (ordinary) arc connecting $v$ and the puncture $P$. Then $\iota(\beta)$ is obtained by tagging $r$ plain at $v$ and notched at $P$. Otherwise, $\iota(\beta)$ is simply $\beta$ with both endpoints tagged plain. See Figure 1(a)

Theorem 2.6 ([FST08, Theorem 7.11, Example 6.7]) Let $T$ be a tagged triangulation of $\mathcal{C}_{n}$ and let $\mathcal{A}$ be the cluster algebra associated to $T$. Then the tagged triangulations of $\mathcal{C}_{n}$ are in bijection with the unlabeled seeds of $\mathcal{A}$, and the tagged arcs $\gamma$ of $\mathcal{C}_{n}$ are in bijection with the cluster variables $x_{\gamma}$, and so a multi-tagged triangulation $\Gamma$ corresponds to a cluster monomial $x_{\Gamma}$ (see Definition 4.1).

Notation 2.7 If $\gamma$ is a tagged arc, let $\gamma^{(p)}$ denote the arc obtained from $\gamma$ by changing the notching at the puncture ${ }_{P}$. If $T$ is a tagged triangulation, let $T^{(p)}$ denote the tagged triangulation that is obtained from $T$ by replacing each $\gamma \in T$ by $\gamma^{(p)}$.

If $\ell$ is an $\ell$-loop enclosing a radius $r$, denote $x_{\ell}:=x_{r} x_{r(p)}$. If $\beta$ is a boundary edge, set $x_{\beta}:=1$.

Remark 2.8 Due to [MSW11. Proposition 3.15], it is enough to work with only two types of tagged triangulations T: one where $T$ has all plain-tagged radii (so that $T^{\circ}$ has a local wheel-like triangulation as in Figure $1(b)$, and one where $T$ has two parallel radii, one tagged plain and the other tagged notched at the puncture (so that $T^{\circ}$ has a self-folded triangle as in Figure $1(c)$ ). 


\section{$3\left(T^{o}, \gamma\right)$-path expansion formula}

We extend Schiffler and Thomas' work [Sch10, ST09] to cover ordinary arcs in a once-punctured disk $\mathcal{C}_{n}$. Following [Sch10, Section 3], we will use the following setup throughout the rest of the paper.

- Let $S$ be an unpunctured surface or $S=\mathcal{C}_{n}$. Let $T^{o}$ be an ideal triangulation of $S$ and let $\gamma \notin T^{o}$ be an ordinary arc of $S$. Recall that an $\ell$-loop is considered an ordinary arc.

- Choose an orientation on $\gamma$, and let $s$ and $t$ be the starting point and the finishing point of $\gamma$. Denote by

$$
s=p_{0}, p_{1}, p_{2}, \ldots, p_{d+1}=t
$$

the points of intersection of $\gamma$ and $T^{o}$ in order. Since $\gamma$ is considered up to homotopy, we pick a representative so that $d$ is minimal. Let $i_{1}, i_{2}, \ldots, i_{d}$ be such that $\tau_{i_{k}}$ is the $\operatorname{arc}$ of $T^{o}$ containing $p_{k}$. See Figure 2(a) where $\tau_{i_{k}}(k=1, \ldots, 5)$ are labeled 1,2, $\ell, r$ and $\ell$.

- For $k=0,1, \ldots, d$, let $\gamma_{k}$ denote the segment of $\gamma$ between the points $p_{k}$ and $p_{k+1}$, and let $\triangle_{k}$ denote the (unique) ideal triangle of $T^{o}$ that $\gamma_{k}$ crosses.

- For $k=1, \ldots, d-1$, define arc $\tau_{\left[\gamma_{k}\right]}$ to be

$$
\tau_{\left[\gamma_{k}\right]}= \begin{cases}\text { the } 3 \text { rd arc in } \triangle_{k} & \text { if } \triangle_{k} \text { is not self-folded } \\ \text { the radius in } \triangle_{k} & \text { if } \triangle_{k} \text { is self-folded. }\end{cases}
$$

Denote the side of $\triangle_{0}$ that lies clockwise of $\tau_{i_{1}}$ by $\tau_{\left[\gamma_{0}\right]}$ and the other side by $\tau_{\left[\gamma_{-1}\right]}$. Similarly, denote the side of $\triangle_{d}$ that lies clockwise of $\tau_{i d}$ by $\tau_{\left[\gamma_{d}\right]}$ and the other side by $\tau_{\left[\gamma_{d+1}\right]}$. If $\triangle_{0}$ (respectively, $\triangle_{d}$ ) is self-folded, then $\tau_{\left[\gamma_{0}\right]}=\tau_{\left[\gamma_{-1}\right]}$ (respectively, $\tau_{\left[\gamma_{d}\right]}=\tau_{\left[\gamma_{d+1}\right]}$ ) is the radius. In Figure 2(a), $\tau_{\left[\gamma_{0}\right]}=b_{1}, \tau_{\left[\gamma_{-1}\right]}=b_{4}, \tau_{\left[\gamma_{d}\right]}=2, \tau_{\left[\gamma_{d+1}\right]}=b_{3}$.

Definition 3.1 (Quasi-arc) If $\tau$ is an ordinary radius of $\mathcal{C}_{n}$ between a marked point $v$ on the boundary and the puncture ${ }_{P}$, let an associated quasi-arc $\tau^{\prime}$ be a curve (not passing through ${ }_{P}$ ) which satisfies the following:

1. $\tau^{\prime}$ is between $v$ and a (non-marked) point $P^{\prime}$ in the vicinity of $P$. (Note that another quasi-arc associated to $\tau$ incident to $P$ may use a different point $P^{\prime \prime}$.)

2. $\tau^{\prime}$ agrees with the arc $\tau$ outside of a radius- $\epsilon$ ball around $P$.

If $\tau$ is a peripheral arc, we let the associated quasi-arc be $\tau$ itself. We label a quasi-arc with the label of the arc that it is associated to.

Definition 3.2 (Extension of complete $\left(T^{o}, \gamma\right)$-path from [Sch10, Definition 2], [MS10, Section 4.1]) $A T^{o}$-path $w=\left(w_{1}, w_{2}, \ldots, w_{\text {length }(w)}\right)$ is a concatenation of steps, oriented quasi-arcs and boundary edges of the ideal triangulation $T^{o}$ of $S$, such that the starting point of a step $w_{i}$ is the finishing point of the previous step $w_{i-1}$. We say that $w$ is a complete $\left(T^{o}, \gamma\right)$-path if the following axioms hold:

(T1) Each even step $w_{2 k}(k=1, \ldots, d)$ is a quasi-arc associated to arc $\tau_{i_{k}}$. Recall that $\tau_{i_{1}}, \ldots, \tau_{i_{d}}$ is the sequence of arcs crossed by $\gamma$ in order. 
(T2) For $k=1,2, \ldots, d-1$, let $\left[p_{k}, p_{k+1}\right]_{w}$ denote the segment of $w$ starting at the point $p_{k}$ following $w_{2 k}$, continuing along $w_{2 k+1}$ and $w_{2(k+1)}$ until the point $p_{k+1}$. Then the segment $\gamma_{k}$ is homotopic to $\left[p_{k}, p_{k+1}\right]_{w}$. If $S=\mathcal{C}_{n}$, then we mean homotopy in the disk minus the puncture. In addition,

- The segment $\gamma_{0}$ is homotopic to the segment $\left[s, p_{1}\right]_{w}$ of the path starting at the point $s=p_{0}$ following $w_{1}$ and $w_{2}$ until the point $p_{1}$;

- The segment $\gamma_{d}$ is homotopic to the segment $\left[p_{d}, t\right]_{w}$ of the path starting at the point $p_{d}$ following $w_{2 d}$ and $w_{2 d+1}$ until the point $p_{d+1}=t$;

It is clear that this definition agrees with the complete $T$-paths of [Sch10, $\overline{\text { MS10] }}$ for unpunctured surfaces. For short, we will refer to a complete $\left(T^{o}, \gamma\right)$-path as simply a $\left(T^{o}, \gamma\right)$-path (or a $T^{o}$-path) for the rest of this paper. By abuse of notation, whenever we say we are going along an arc or a side of an ideal triangle $\triangle_{k}$ (as part of a $T^{o}$-path), we mean traversing an associated quasi-arc. Note that except for the case when $\tau$ is a radius, no abuse of notation is actually needed.

Remark 3.3 We collect the following observations regarding conditions (T1) and (T2).

- By (T1), the length $\ell(w)$ of $w$ is always $2 d+1$, where $d$ is the number of times that $\gamma$ intersects $T^{o}$.

- It is possible to have $w_{k}=w_{k+1}$ and, if $k$ is odd, to have $w_{k}=w_{k+1}=w_{k+2}$. However, since $w_{2 k}=\tau_{i_{k}}$ and $w_{2(k+1)}=\tau_{i_{k+1}}$ by (T1) but $\tau_{i_{k}} \neq \tau_{i_{k+1}}$, no more than three consecutive steps can coincide.

- The homotopy condition of (T2) requires each $w_{2 k+1}(k=0, \ldots, d)$ to traverse a side of the ideal triangle $\triangle_{k}$.

Definition 3.4 (Laurent monomial from a $T^{o}$-path) We identify each step with the label of the quasiarc/boundary edge which it traverses and define the Laurent monomial $x(w)$ corresponding to a complete $\left(T^{o}, \gamma\right)$-path $w$ by

$$
x(w)=\prod_{i \text { odd }} x_{w_{i}} \prod_{i \text { even }} x_{w_{i}}^{-1}
$$

Remark 3.5 Two or more $\left(T^{o}, \gamma\right)$-paths may correspond to the same Laurent monomial, e.g. see Example 3.12. For each $\left(T^{o}, \gamma\right)$-path $w$, the denominator of $x(w)$, before reducing, is equal to $x_{i_{1}}, x_{i_{2}}, \ldots, x_{i_{d}}$ which corresponds to the arcs $\tau_{i_{1}}, \ldots, \tau_{i_{d}}$ of $T^{o}$ which cross $\gamma$.

Definition 3.6 (Backtrack, non-backtrack cycle) Let $w$ be a $T^{o}$-path, let $\tau$ be an arc of $T^{o}$, and let $\left(w_{k}, w_{k+1}\right)$ be a pair of consecutive steps going along $\tau$.

- If $\tau$ is a peripheral arc, we say that $\left(w_{k}, w_{k+1}\right)$ is a backtrack if it is contractible (equivalently, if $w_{k}$ and $w_{k+1}$ are opposite orientations of $\left.\tau\right)$.

- If $\tau$ is a radius between the puncture ${ }_{P}$ and a marked point $v$ on the boundary, then we say that $\left(w_{k}, w_{k+1}\right)$ is a backtrack if one of the following holds:

1. $w_{k}$ ends at $v$, so that $\left(w_{k}, w_{k+1}\right)$ is a (non-cyclic) concatenation of two associated quasi-arcs $P^{\prime} \leadsto v \leadsto P^{\prime \prime}$ where $P^{\prime}$ and $P^{\prime \prime}$ are in the vicinity of the puncture (Figure 5(a)). 


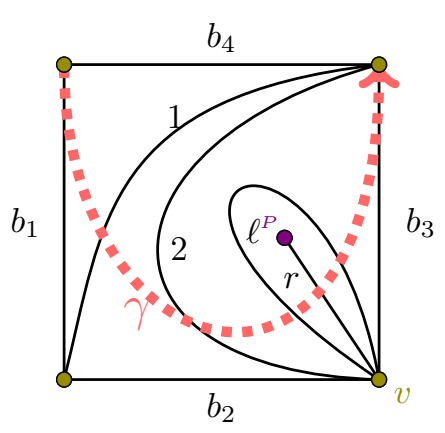

(a) $\operatorname{Arc} \gamma$ of $\mathcal{C}_{4}$ where $d=5$.

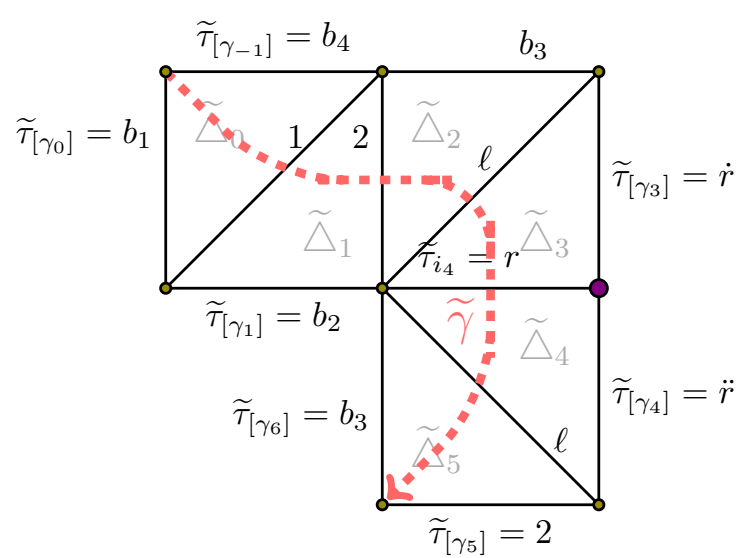

(b) Lifted arc $\widetilde{\gamma}$ on the constructed triangulated $(5+3)$-gon $\widetilde{S_{\gamma}}$.

Fig. 2: The construction of the triangulated polygon $\widetilde{S_{\gamma}}$ and lifted $\operatorname{arc} \widetilde{\gamma}$ for an $\operatorname{arc} \gamma$ of $\mathcal{C}_{n}$.

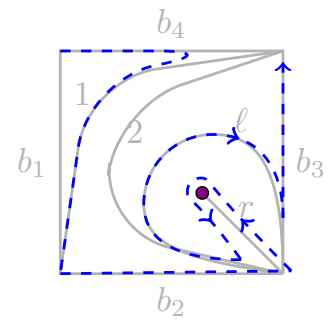

(a) $\left(b_{4}, 1, b_{2}, \mathbf{r}, \mathbf{r}, \ell, b_{3}\right)$

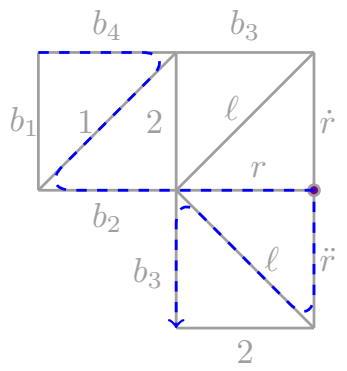

(e) $\left(b_{4}, 1, b_{2}, \mathbf{r}, \ddot{\mathbf{r}}, \ell, b_{3}\right)$

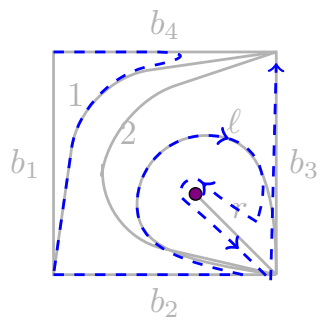

(b) $\left(b_{4}, 1, b_{2}, \ell, \mathbf{r}, \mathbf{r}, b_{3}\right)$

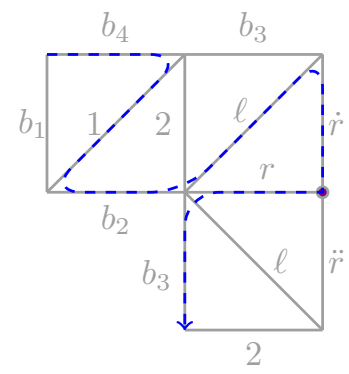

(f) $\left(b_{4}, 1, b_{2}, \ell, \dot{\mathbf{r}}, \mathbf{r}, b_{3}\right)$

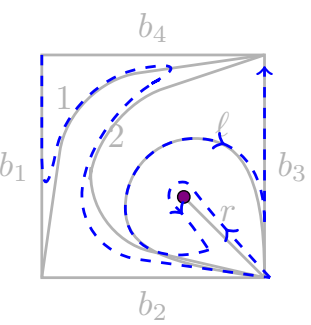

(c) $\left(b_{1}, 1,2, \mathbf{r}, \mathbf{r}, \ell, b_{3}\right)$

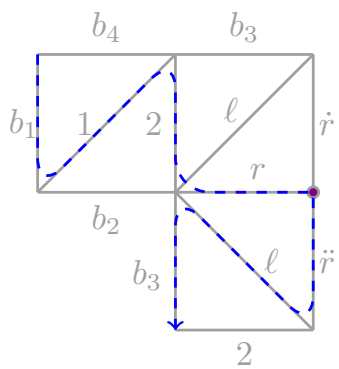

(g) $\left(b_{1}, 1,2, \mathbf{r}, \ddot{\mathbf{r}}, \ell, b_{3}\right)$

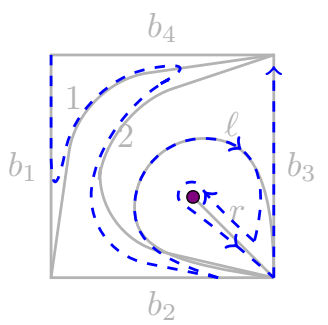

(d) $\left(b_{1}, 1,2, \ell, \mathbf{r}, \mathbf{r}, b_{3}\right)$

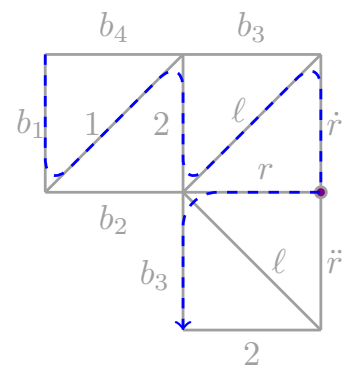

(h) $\left(b_{1}, 1,2, \ell, \dot{\mathbf{r}}, \mathbf{r}, b_{3}\right)$

Fig. 3: The four $\left(T^{o}, \gamma\right)$-paths (and their lifts) which contain a (counterclockwise) non-backtrack $r, r$. All backtracks have been omitted, and steps are not drawn exactly along the arcs/boundary edges for illustration purposes.
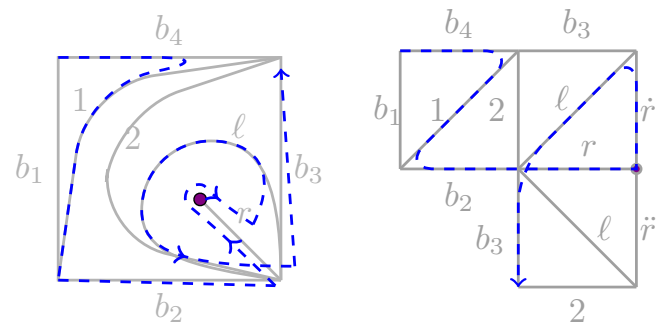

Fig. 4: A non- $\left(T^{o}, \gamma\right)$-path (and its lift on $\left.\widetilde{S_{\gamma}}\right)$ that goes along the same arcs as the $\left(T^{o}, \gamma\right)$-path of Figure 3(a) 
2. $\left(w_{k}, w_{k+1}\right)$ is a contractible concatenation of two associated quasi-arcs $v \leadsto P^{\prime} \leadsto v$ where $P^{\prime}$ is in the vicinity of the puncture (Figure $5(b)$ ).

- Otherwise, $\left(w_{k}, w_{k+1}\right)$ is homotopic to a non-contractible loop starting and ending at at a marked point $v$ on the boundary, and we say that $\left(w_{k}, w_{k+1}\right)$ is a non-backtrack cycle.

- Two special non-backtrack cycles are as follows. Let $\tau$ be a radius and let $\left(w_{k}, w_{k+1}\right)$ start at the boundary and goes around the puncture exactly once. We say that $\left(w_{k}, w_{k+1}\right)$ is a counterclockwise non-backtrack if it goes counterclockwise (Figure 5(c)), and a clockwise non-backtrack if it goes clockwise (Figure $5(d)$ ).

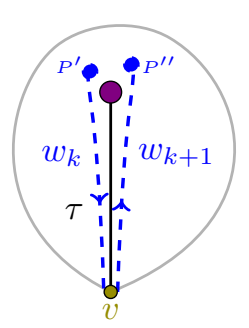

(a) Non-cyclic backtrack.

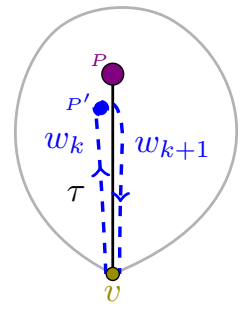

(b) Backtrack.

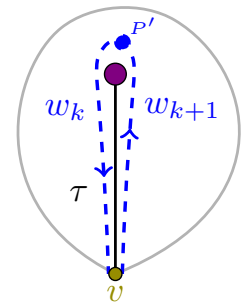

(c)

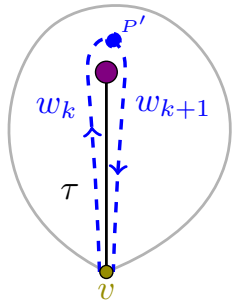

(d) Clockwise non-backtrack.

Counterclockwise non-backtrack.

Fig. 5: The four possibilities of a pair $\left(w_{k}, w_{k+1}\right)$ of consecutive steps along a radius $\tau$.

Note that Figure 5 illustrates all four possibilities for a pair $\left(w_{k}, w_{k+1}\right)$ along a radius.

\section{Definition 3.7}

We say that $\gamma$ crosses the radius of a self-folded triangle in the counterclockwise direction (respectively, clockwise direction) if it matches (respectively, if $\gamma$ has the opposite orientation of) Figure 2(a)

Lemma 3.8 Let $T^{o}$ be an ideal triangulation and let $w$ be a $\left(T^{o}, \gamma\right)$-path containing a non-backtrack cycle $\left(w_{k}, w_{k+1}\right)$. Then $\left(w_{k}, w_{k+1}\right)$ must go along associated quasi-arcs of the radius $r$ of a self-folded triangle (and hence, $T^{o}$ contains a self-folded triangle, and $\gamma$ crosses $r$ ). Furthermore, the orientation of $\gamma$ as it crosses $r$ determines whether $\left(w_{k}, w_{k+1}\right)$ is a clockwise or counterclockwise non-backtrack.

Proposition $3.9 A\left(T^{o}, \gamma\right)$-path $w$ is uniquely determined by its sequence of labels $w_{1}, \ldots, w_{2 d+1}$, forgetting the orientations of the steps and whether a consecutive pair is a non-backtrack or a backtrack.

Remark 3.10 To illustrate Proposition 3.9. consider the $\left(T^{o}, \gamma\right)$-path of Figure $3(a)$

$$
w=\left(b_{4}, 1, b_{2}, 2,2, \ell, \ell, r, r, \ell, b_{3}\right) .
$$

The pair $\left(w_{8}, w_{9}\right)=(r, r)$ is a counterclockwise non-backtrack along the radius $r$, and $w_{10}$ goes clockwise around $\ell$. Consider a different path $w^{\prime}$ (see Figure 4) which goes along the same sequence of arcs such that $\left(w_{8}^{\prime}, w_{9}^{\prime}\right)$ is a clockwise non-backtrack along the radius, and $w_{10}^{\prime}$ goes counterclockwise around $\ell$. Even though $w^{\prime}$ is homotopic to $\gamma$, the segment $\gamma_{4}$ is not homotopic to $\left[p_{4}, p_{5}\right]_{w}$, which violates Definition $3.2(T 2)$. 
Theorem 3.11 ( $T^{o}$-path formula for $\mathcal{C}_{n}$, an extension of [Sch10, Theorem 3.1], [ST09, Theorem 3.2]) Let $T^{o}$ be an ideal triangulation of $\mathcal{C}_{n}$, let $\gamma \notin T^{o}$ be an ordinary arc of $\mathcal{C}_{n}$, and let $x_{\gamma}$ denote the corresponding element in the cluster algebra which arises from $\mathcal{C}_{n}$. Then

$$
x_{\gamma}=\sum_{w} x(w)
$$

where the sum is taken over all $\left(T^{o}, \gamma\right)$-paths. The formula does not depend on the choice of orientation on $\gamma$.

Example 3.12 The nine $\left(T^{o}, \gamma\right)$-paths from Figure 2(a) are as follows (with the backtracks underlined):
(1.) $\left(b_{4}, 1, b_{2}, 2, b_{3}, \ell, \underline{r, r}, \underline{\ell, \ell}, b_{3}\right)$
(6.) $\left(b_{4}, 1, b_{2}, \underline{2,2}, \underline{\left.\ell, \ell, \mathbf{r}, \mathbf{r}, \ell, b_{3}\right)}\right.$
(2.) $\left(b_{4}, \underline{1,1}, 2, \ell, \ell, \underline{r, r}, \underline{\ell, \ell}, b_{3}\right)$
(3.) $\left(b_{1}, 1,2,2,2, \underline{\ell, \ell}, \underline{r, r}, \ell, 2\right)$
(7.) $\left(b_{4}, 1, b_{2}, \underline{2,2}, \ell, \mathbf{r}, \mathbf{r}, \underline{\left.\ell, \ell, b_{3}\right)}\right.$
(4.) $\left(b_{1}, 1, \underline{2,2}, b_{3}, \ell, \underline{r, r}, \underline{\ell, \ell}, b_{3}\right)$
(8.) $\left(b_{1}, 1, \underline{2,2}, 2, \underline{\ell, \ell}, \mathbf{r}, \mathbf{r}, \ell, b_{3}\right)$
(5.) $\left(b_{4}, 1, b_{2}, \underline{2,2}, \underline{\ell, \ell}, \underline{r, r}, \ell, 2\right)$
(9.) $\left(b_{1}, 1, \underline{2,2}, 2, \ell, \mathbf{r}, \mathbf{r}, \underline{\ell, \ell}, b_{3}\right)$

The last four of these $\left(T^{o}, \gamma\right)$-paths contain a (counterclockwise) non-backtrack $(r, r)$ and are illustrated in Figure 3 They are drawn so that the backtracks are ignored.

We apply Theorem 3.11 and replace each $\left(x_{r} x_{\ell}\right) /\left(x_{\ell} x_{r} x_{\ell}\right)$ with $1 / x_{\ell}$ and each $x_{\ell}$ with $x_{r} x_{r^{(p)}}$ to get

$$
\begin{aligned}
x_{\gamma}= & \left(x_{b_{4}} x_{b_{2}} x_{b_{3}} x_{b_{3}}+x_{b_{4}} x_{1} x_{r} x_{r(p)} x_{b_{3}}+\right. \\
& x_{b_{1}} x_{2}^{3}+x_{b_{1}} x_{b_{3}}^{2} x_{2}+x_{b_{4}} x_{b_{2}} x_{2}^{2}+ \\
& \left.2 x_{b_{4}} x_{b_{2}} x_{b_{3}} x_{2}+2 x_{b_{1}} x_{b_{3}} x_{2}^{2}\right) \\
& /\left(x_{1} x_{2} x_{r} x_{r(p)}\right)
\end{aligned}
$$

where $x_{b_{j}}=1$ for each boundary edge $b_{j}$.

Proof of Theorem 3.11 (sketch): For full details, see [GM14]. Let $S_{\gamma}$ be the union of the ideal triangles $\triangle_{k}(k=0, \ldots, d)$ crossed by $\gamma$. Following [MSW11, Section 7], we construct a triangulated $(d+3)$-gon $\widetilde{S_{\gamma}}$ that is modeled after $S_{\gamma}$ so that the internal edges $\widetilde{\tau}_{i_{1}}, \widetilde{\tau}_{i_{2}}, \ldots, \widetilde{\tau}_{i_{d}}$ of $\widetilde{S_{\gamma}}$ have the same labels as $\tau_{i_{1}}$, $\tau_{i_{2}}, \ldots, \tau_{i_{d}}$, and the boundary edges $\widetilde{\tau}_{\left[\gamma_{-1}\right]}, \widetilde{\tau}_{\left[\gamma_{0}\right]}, \widetilde{\tau}_{\left[\gamma_{1}\right]}, \widetilde{\tau}_{\left[\gamma_{2}\right]}, \ldots, \widetilde{\tau}_{\left[\gamma_{d-1}\right]}, \widetilde{\tau}_{\left[\gamma_{d}\right]}, \widetilde{\tau}_{\left[\gamma_{d+1}\right]}$ of $\widetilde{S}_{\gamma}$ correspond to arcs or boundary edges $\tau_{\left[\gamma_{-1}\right]}, \tau_{\left[\gamma_{0}\right]}, \tau_{\left[\gamma_{1}\right]}, \tau_{\left[\gamma_{2}\right]}, \ldots, \tau_{\left[\gamma_{d-1}\right]}, \tau_{\left[\gamma_{d}\right]}, \tau_{\left[\gamma_{d+1}\right]}$ belonging to $S_{\gamma}$. Every triangle $\widetilde{\triangle}_{k}$ in $\widetilde{S_{\gamma}}$ corresponds to an ideal triangle in $T^{o}$, and their orientations agree. When $\triangle_{k}$ is a self-folded triangle with radius $r$ and $\ell$-loop $\ell$, its lift $\widetilde{\triangle}_{k}$ is formed by two lifts of $r$ and a lift of $\ell$. We construct the lift $\widetilde{\gamma}$ of $\gamma$ in a similar way. Figure 2(b) illustrates the lift $\widetilde{\gamma}$ of $\gamma$ on the lift $\widetilde{S_{\gamma}}$ of $S_{\gamma}$ of Figure 2(a).

Step 1: We prove that the covering map $\pi: \widetilde{S_{\gamma}} \rightarrow S_{\gamma}$ induces a bijection

$$
\begin{aligned}
\bar{\pi}:\left\{\left(\widetilde{S_{\gamma}}, \widetilde{\gamma}\right) \text {-paths on } \widetilde{S_{\gamma}}\right\} & \rightarrow\left\{\left(T^{o}, \gamma\right) \text {-paths on } S_{\gamma}\right\} \\
\bar{\pi}\left(\widetilde{w}_{1}, \widetilde{w}_{2}, \ldots, \widetilde{w}_{2 d+1}\right) & =\left(\pi\left(\widetilde{w}_{1}\right), \pi\left(\widetilde{w}_{2}\right), \ldots, \pi\left(\widetilde{w}_{2 d+1}\right)\right) .
\end{aligned}
$$


We observe that a pair $\left(\widetilde{w}_{k}, \widetilde{w}_{k+1}\right)$ from a $\left(\widetilde{S_{\gamma}}, \widetilde{\gamma}\right)$-path $\widetilde{w}$ goes along the same edge on $\widetilde{S_{\gamma}}$ if and only if their image $\left(w_{k}, w_{k+1}\right)$ is a backtrack. For example, in Figure 3 , every pair $(\dot{r}, r)$ or $(r, \ddot{r})$ on $\widetilde{S_{\gamma}}$ corresponds to a (counterclockwise) non-backtrack $(r, r)$ on $\mathcal{C}_{n}$.

Step 2: [MS10, Lemma 4.5] gives a bijection $F$ from the perfect matchings of the snake graph $G_{T^{o}, \gamma}$ onto the $\left(\widetilde{S_{\gamma}}, \widetilde{\gamma}\right)$-paths on $\widetilde{S_{\gamma}}$. Certain diagonals in $G_{T^{o}, \gamma}$ correspond to the arcs $\tau_{i_{k}}$ crossed by $\gamma$, and these correspond to the even steps of each $\left(\widetilde{S_{\gamma}}, \widetilde{\gamma}\right)$-path. For each matching $E$ of $G_{T^{o}, \gamma}$, the matched edges correspond to the odd steps of the $\left(\widetilde{S_{\gamma}}, \widetilde{\gamma}\right)$-path $F(E)$.

Step 3: The bijections $\bar{\pi}$ and $F$ compose to form a bijection between $\left(T^{o}, \gamma\right)$-paths on $\mathcal{C}_{n}$ and perfect matchings of the snake graph $G_{T^{\circ}, \gamma}$.

Step 4: Per [MSW11, Theorem 4.10], the $T^{o}$-Laurent expansion of $x_{\gamma}$ is given by

$$
x_{\gamma}=\sum_{E} \frac{x(E)}{\operatorname{cross}\left(T^{o}, \gamma\right)}
$$

where the sum is over all perfect matchings $E$ of $G_{T^{o}, \gamma}$ and

$$
\operatorname{cross}\left(T^{o}, \gamma\right)=\prod_{k=1}^{d} x_{\tau_{i_{k}}} .
$$

\section{Atomic Bases}

\subsection{Combinatorial proof of atomic bases for type $D$}

Definition 4.1 (Cluster monomials) Let $\mathcal{A}$ be a skew-symmetric cluster algebra. A cluster monomial is a monomial in cluster variables all belonging to a single cluster.

Recall that, in the case that $\mathcal{A}$ arises from a surface, a cluster corresponds to a tagged triangulation and a cluster monomial corresponds to a multi-tagged triangulation. See Definition 2.4 for a list of compatible pairs of tagged arcs of $\mathcal{C}_{n}$.

The concepts of positive elements and atomic bases were first introduced in [SZ04] for the case of an annulus with one marked point on each boundary.

Definition 4.2 (Positive elements and atomic bases) An element $y \in \mathcal{A}$ is called positive if the Laurent expansion of $y$ in the variables of every cluster of $\mathcal{A}$ has non-negative coefficients.

$A \mathbb{Z}$-linear basis $\mathcal{B}$ of $\mathcal{A}$ is called an atomic basis of $\mathcal{A}$ if any positive element of $\mathcal{A}$ is a non-negative $\mathbb{Z}$-linear combination of $\mathcal{B}$. Note that, if such a $\mathcal{B}$ exists, $\mathcal{B}$ is the collection of all indecomposable positive elements (i.e. elements which cannot be written as a sum of positive elements) of $\mathcal{A}$, hence it is unique.

Remark 4.3 To prove that a collection $\mathcal{B} \subset \mathcal{A}$ is an atomic basis of $\mathcal{A}$, it suffices to verify the following:

Every element of $\mathcal{B}$ is a positive element of $\mathcal{A}$.

$\mathcal{B}$ is a $\mathbb{Z}$-linear basis of $\mathcal{A}$.

Every positive element $y \in \mathcal{A}$ can be written as a $\mathbb{Z}_{\geq 0}$-linear combination of $\mathcal{B}$. 
Theorem 4.4 ([Cer11]) If $\mathcal{A}$ is a cluster algebra of type $A, D$, or $E$, the cluster monomials of $\mathcal{A}$ form the atomic basis of $\mathcal{A}$.

Proof: [HL10, Nak11], [CK08], and [Cer11, CL12] give representation theoretic proofs for [4.1], 44.2], and (4.3), respectively. [MSW11] and [MSW13] provide combinatorial arguments for 4.1] and (4.2), respectively, and [DT13] gives a combinatorial proof for the type $A$ for [4.3). We present in [GM14] a combinatorial proof of (4.3) which relies on the $T^{\circ}$-path formula (Theorem 3.11) and is inspired by [DT13].

Recall that a proper Laurent monomial in variables $u_{i}$ is a product of the form $u_{1}^{c_{1}} \cdots u_{r}^{c_{r}}$ where at least one of the $c_{i}$ is negative. Note that, by the same logic used in [SZ04, CL12, DT13], if $\mathcal{A}$ is a cluster algebra of type $D$ and $\mathcal{B}$ is the set of cluster monomials and if (4.1) and (4.2) are assumed, then (4.3) is a direct consequence of the following.

Lemma 4.5 Consider a once-punctured polygon $\mathcal{C}_{n}$. For every tagged triangulation $T$ and every multitagged triangulation $\Sigma$ which is not compatible with $T$, the T-expansion of $x_{\Sigma}$ is a sum of proper Laurent monomials.

Remark 4.6 A general version of Lemma 4.5 is known as the proper Laurent monomial property (following [CL12]) which was proven for cluster algebras from surfaces in [CL12] and then for any skewsymmetric cluster algebras in [CKLP13], using representation theoretic arguments in both cases.

Proof (sketch): Suppose $x_{\Sigma}$ is a cluster monomial not compatible with a tagged triangulation $T$, i.e. $\Sigma$ contains an arc $\sigma$ which is not in $T$. For brevity, suppose that all tagged arcs of $\Sigma \backslash T$ are peripheral and the corresponding ideal triangulation $T^{o}$ has no self-folded triangle. For full details and the rest of the cases, see [GM14].

Step 1: We choose a tagged arc $\sigma \in \Sigma \backslash T$ such that $\sigma$ is as close as possible to the puncture and, if possible, $\sigma$ only crosses every arc of $T$ at most once. Let $(T, \sigma)$-cross (respectively, $(T, \sigma)$-doublecross) be the set of arcs of $T$ which $\sigma$ crosses (respectively, the set of arcs of $T$ which $\sigma$ crosses twice).

Step 2: For each $(T, \sigma)$-path $w$, we compare the number of odd steps (contributing to the numerator of $x(w)$ ) and the number of even steps (contributing to the denominator of $x(w)$ ) that belong to $(T, \sigma)$-cross and $(T, \sigma)$-doublecross. This allows us to show that each term in the $T$-expansion of $x_{\sigma}$ is of negative degree either with respect to the cluster variables corresponding to $(T, \sigma)$-cross or with respect to the cluster variables corresponding to $(T, \sigma)$-doublecross.

Step 3: Similarly, for each factor $x_{\beta}$ in the product of $x_{\Sigma}$, we consider a $(T, \beta)$-path and compare the number of odd steps versus the number of even steps to show that every term in the $T$-expansion of $x_{\beta}$ has non-positive degree with respect to both the cluster variables corresponding to $(T, \sigma)$-cross and the cluster variables corresponding to $(T, \sigma)$-doublecross.

Step 4: It follows, since $\sigma \in \Sigma$ and every term in the $T$-expansion of $x_{\Sigma}$ has non-positive degree with respect to both $(T, \sigma)$-cross and $(T, \sigma)$-doublecross, that every term in the $T$-expansion of $x_{\Sigma}$ has negative degree with respect to either $(T, \sigma)$-cross or $(T, \sigma)$-doublecross. 


\subsection{Further directions}

It is not known for a general cluster algebra whether its set of indecomposable positive elements forms an atomic basis. Examples of cluster algebras where this fails to happen are those of rank 2 where the exchange matrix looks like $\left(\begin{array}{cc}0 & b \\ -c & 0\end{array}\right)$ with $b c \geq 5$ [LLZ14].

Per [MSW13, Conjecture 1.5], we are exploring the existence of atomic bases for type $\widetilde{D}_{n-1}$ cluster algebras (which arise from $(n-3)$-gons with 2 punctures). Toward this goal, we would like to extend the $T^{o}$-path expansion formula to general bordered surfaces with more than 1 puncture. Additionally, the $T^{o}$ path formula presented in this paper was only done for ordinary arcs in the setting of ideal triangulations. It would be desirable to prove a similar formula for tagged arcs in the setting of tagged triangulations.

\section{Acknowledgements}

The authors would like to thank Pasha Pylyavskyy, Vic Reiner, and Peter Webb for looking over an early version of this paper, Hugh Thomas for helpful discussions, and the referees for many useful comments. The authors were supported by NSF Grants DMS-1067183 and DMS-1148634. This paper is an abridged version of [GM14].

\section{References}

[BM09] Karin Baur and Robert J. Marsh. Frieze patterns for punctured discs. J. Algebraic Combin., 30(3):349-379, 2009.

[Cer11] Giovanni Cerulli Irelli. Positivity in skew-symmetric cluster algebras of finite type. arXiv preprint arXiv:1102.3050, 2011.

[CKLP13] Giovanni Cerulli Irelli, Bernhard Keller, Daniel Labardini-Fragoso, and Pierre-Guy Plamondon. Linear independence of cluster monomials for skew-symmetric cluster algebras. Compos. Math., 149(10):1753-1764, 2013.

[CL12] Giovanni Cerulli Irelli and Daniel Labardini-Fragoso. Quivers with potentials associated to triangulated surfaces, Part III: tagged triangulations and cluster monomials. Compos. Math., 148(6):1833-1866, 2012.

[CK08] Philippe Caldero and Bernhard Keller. From triangulated categories to cluster algebras. Invent. Math., 172(1):169-211, 2008.

[CP14] Cesar Ceballos and Vincent Pilaud. Cluster algebras of type D: Pseudotriangulations approach. In preparation, 2014.

[DT13] Grégoire Dupont and Hugh Thomas. Atomic bases of cluster algebras of types $A$ and $\tilde{A}$. Proc. Lond. Math. Soc. (3), 107(4):825-850, 2013.

[FG06] Vladimir Fock and Alexander Goncharov. Moduli spaces of local systems and higher Teichmüller theory. Publ. Math. Inst. Hautes Études Sci., (103):1-211, 2006. 
[FG09] Vladimir V. Fock and Alexander B. Goncharov. Cluster ensembles, quantization and the dilogarithm. Ann. Sci. Éc. Norm. Supér. (4), 42(6):865-930, 2009.

[FST08] Sergey Fomin, Michael Shapiro, and Dylan Thurston. Cluster algebras and triangulated surfaces. I. Cluster complexes. Acta Math., 201(1):83-146, 2008.

[FZ02] Sergey Fomin and Andrei Zelevinsky. Cluster algebras I: Foundations. J. Amer. Math. Soc., 15(2):497-529 (electronic), 2002.

[FZ03] Sergey Fomin and Andrei Zelevinsky. Cluster algebras II: Finite type classification. Invent. Math., 154(1):63-121, 2003.

[GM14] Emily Gunawan and Gregg Musiker. T-path formula and atomic bases for cluster algebras of type D. arXiv preprint arXiv:1409.3610, 2014.

[GSV05] Michael Gekhtman, Michael Shapiro, and Alek Vainshtein. Cluster algebras and WeilPetersson forms. Duke Math. J., 127(2):291-311, 2005.

[HL10] David Hernandez and Bernard Leclerc. Cluster algebras and quantum affine algebras. Duke Math. J., 154(2):265-341, 2010.

[LLZ14] Kyungyong Lee, Li Li, and Andrei Zelevinsky. Positivity and tameness in rank 2 cluster algebras. J. Algebraic Combin., 40(3):823-840, 2014.

[MS10] Gregg Musiker and Ralf Schiffler. Cluster expansion formulas and perfect matchings. $J$. Algebraic Combin., 32(2):187-209, 2010.

[MSW11] Gregg Musiker, Ralf Schiffler, and Lauren Williams. Positivity for cluster algebras from surfaces. Adv. Math., 227(6):2241-2308, 2011.

[MSW13] Gregg Musiker, Ralf Schiffler, and Lauren Williams. Bases for cluster algebras from surfaces. Compos. Math., 149(2):217-263, 2013.

[Nak11] Hiraku Nakajima. Quiver varieties and cluster algebras. Kyoto J. Math., 51(1):71-126, 2011.

[Sch08] Ralf Schiffler. A geometric model for cluster categories of type $D_{n}$. J. Algebraic Combin., 27(1):1-21, 2008.

[Sch10] Ralf Schiffler. On cluster algebras arising from unpunctured surfaces II. Adv. Math., 223(6):1885-1923, 2010.

[ST09] Ralf Schiffler and Hugh Thomas. On cluster algebras arising from unpunctured surfaces. Int. Math. Res. Not. IMRN, (17):3160-3189, 2009.

[SZ04] Paul Sherman and Andrei Zelevinsky. Positivity and canonical bases in rank 2 cluster algebras of finite and affine types. Mosc. Math. J., 4(4):947-974, 982, 2004. 